\title{
Kystes inflammatoires des maxillaires : mise au point
}

\section{RÉSUMÉ}

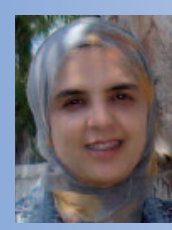

Fouzia HAKKOU

Résidente en odontologie chirurgicale, Service d'odontologie chirurgicale,

Centre de consultation

et de traitement dentaire,

CH Ibn Sina, Rabat.

Faculté de médecine dentaire,

BP 6212,

Rabat-Instituts, Maroc.

\section{Saliha CHBICHEB}

Professeur agrégé

en odontologie chirurgicale,

Service d'odontologie chirurgicale,

Centre de consultation

et de traitement dentaire,

CH Ibn Sina, Rabat.

\section{Imane ACHOUR}

Spécialiste en odontologie conservatrice, Service d'odontologie chirurgicale,

Centre de consultation

et de traitement dentaire,

CH Ibn Sina, Rabat.

\section{Wafae EL WADY}

Professeur de l'enseignement supérieur, Chef de service d'odontologie chirurgicale, Service d'odontologie chirurgicale,

Centre de consultation

et de traitement dentaire,

CH Ibn Sina, Rabat.

Les kystes des maxillaires sont des lésions intraosseuses, d'étiologies variées, possédant une structure histologique particulière caractérisée par la présence d'une enveloppe épithéliale, kératinisée ou non, et un contenu liquide ou semi-liquide. Les kystes inflammatoires sont les plus fréquents parmi les kystes des maxillaires. C'est une variété qui regroupe les kystes radiculaires, résiduels et paradentaires car la croissance de leur paroi épithéliale odontogène est initiée par les produits d'inflammation. Pour chaque type de ces kystes, certaines particularités sont retrouvées. Cet article a pour but de présenter à travers une revue de littérature les aspects cliniques, radiologiques et anatomopathologiques des kystes inflammatoires des maxillaires.

- kystes inflammatoires

- kyste radiculaire

- kyste résiduel

- kyste paradentaire
AOS 2012;260:301-311

DOI: $10.1051 /$ aos/2012402

(C) EDP Sciences 
Selon la classification de l'Organisation mondiale de la santé de 1992, les kystes odontogènes liés à une inflammation comprennent le kyste apical, kyste latéroradiculaire, le kyste résiduel et le kyste paradentaire [1].

\section{Kystes radiculo-dentaires}

Il s'agit de la lésion la plus fréquente des lésions kystiques des maxillaires (55 à $63 \%$ ) [2]. Il semble plus fréquent après la première décade avec un pic lors des $3^{\mathrm{e}}$ et $5^{\mathrm{e}}$ décades et une prédilection chez les hommes. II se localise de façon préférentielle au niveau du secteur antérieur maxillaire ainsi qu'au niveau des molaires [2].

Sur le plan étiopathogénique, les kystes radiculo-dentaires sont des entités cliniques appartenant à la lignée chronique des parodontites apicales. Ces dernières sont définies comme étant des lésions inflammatoires du parodonte profond, périradiculaires, principalement de la région périapicale, consécutives à l'infection bactérienne de l'endodonte [3]. Ces bactéries atteignent la pulpe dentaire à travers de multiples voies de contamination. Ainsi, si la carie dentaire reste la cause la plus fréquente de I'infection endodontique, l'exposition des tubuli dentinaires à l'environnement oral, suite à une fracture amélodentinaire, constitue aussi une voie de contamination potentielle [4]. La flore bactérienne endodontique ainsi établie, colonisant un espace à l'abri des défenses générales de l'organisme, exprime son pouvoir pathogène via les métabolites et les produits de dégradation de certaines espèces [4]. Hormis les exotoxines et les leukotoxines qui agissent sur les membranes et provoquent des petits trous qui entraînent la lyse des leucocytes, ce sont les endotoxines qui sont les plus impliquées dans la genèse des lésions périapicales [4, 5]. À l'extrémité radiculaire, un bouchon formé de leucocytes polymorphonucléaires [6] ou de cellules épithéliales va contenir la grande majorité de ces bactéries à l'intérieur du canal. Néanmoins, cela n'empêche pas les toxines, issues de leur métabolisme, de gagner le périapex, déclenchant et entretenant la réaction inflammatoire.

La présence continue d'irritants intracanalaires favorise graduellement le passage de l'inflammation initiale vers une lésion encapsulée par un tissu conjonctif collagénique contenant de plus en plus de macrophages et lymphocytes, produisant des anticorps et des cytokines. Au cours de cette transformation, les cytokines vont orienter le statut de la lésion, tantôt en stimulant les facteurs d'activation des ostéoclastes et en favorisant la résorption osseuse, tantôt en favorisant les facteurs de croissance stimulant la prolifération des fibroblastes et l'angiogenèse, la reconstruction du conjonctif et le ralentissement de la résorption. Les cellules $T$ Helper de type 1 et 2 sont impliquées dans ce processus inhibiteur de la destruction osseuse. Ainsi, le granulome reflète un stade d'équilibre entre les agresseurs confinés dans le canal et une défense autocontrôlée. Il peut rester au repos et sans symptômes pendant plusieurs années et sans modification radiographique décelable [6].

Histologiquement, le granulome est un tissu de granulation infiltré et vascularisé, limité par une membrane fibro-conjonctive. Cette capsule, bien délimitée, consiste en fibres de collagène denses, fermement attachées à la sur- 
face radiculaire, de telle façon que si l'on extrait la dent, la lésion appendue à l'apex est retirée simultanément. Au sein de ce granulome, les cordons épithéliaux prolifèrent en se dirigeant au hasard et en emprisonnant des îlots vasculaires et conjonctifs infiltrés. $50 \%$ des lésions sont épithélialisées [6]. Cet épithélium se ramifie toujours en direction de la récession osseuse, mais peut aussi s'appliquer largement à la surface radiculaire [7].

Tous les granulomes n'évoluent pas en kyste, mais ces derniers sont considérés comme une séquelle directe des granulomes. Seulement $20 \%$ des lésions épithélialisées seraient des kystes, et, parmi celles-ci, la moitié correspondrait à des kystes en poche s'ouvrant dans le canal et non à des kystes vrais $[4,6]$.

La pathogenèse d'un kyste vrai suppose trois étapes [6] :

- les débris épithéliaux de Malassez quiescents commencent à proliférer, probablement sous I'influence de facteurs de croissance (EGF, PDGF) libérés par les cellules résidentes activées ;

- une cavité bordée par un épithélium se développe. Deux hypothèses sont avancées pour expliquer la formation de cette cavité : la théorie de la déficience nutritive progressive de la partie centrale de la lésion, qui finalement se nécrose puis se liquéfie ; et la théorie de l'encapsulement d'un abcès secondaire par une prolifération épithéliale périphérique ;

- le kyste augmente en volume. Le rôle de la pression osmotique semble négligeable. L'expansion du kyste est plus probablement due à l'activité des agents moléculaires de résorption osseuse, produits par les cellules résidentes du kyste et qui diffusent vers la périphérie osseuse : PGs, cytokines, métalloprotéinases.
Dans le cas des kystes en poche, la formation de la lésion est différente [6] :

- une barrière de neutrophiles s'installe à l'apex pour contenir les germes intracanalaires. Avec le temps, les neutrophiles s'accumulent et forment un bouchon apical ;

- les cordons épithéliaux prolifèrent en périphérie et réalisent un attachement épithélial aux parois radiculaires, isolant le bouchon apical de neutrophiles ;

- le bouchon continu à croître. Lorsqu'il se désintègre, une micro-poche se forme, puis s'élargit formant un diverticule du canal, en forme de sac, confiné à l'intérieur de la lésion. L'expansion de la poche kystique et l'activité de la lésion sont identiques à celles du kyste vrai.

L'accumulation de produits cristallisés dans la cavité kystique et l'organisation spécifique de la lésion elle-même peuvent interférer avec le processus de guérison après thérapeutique endodontique conventionnelle et expliquer la persistance de ces lésions sur des périodes indéterminées.

Sur le plan clinique, le kyste radiculaire se développe $d$ 'une manière asymptomatique. Le taux de croissance d'un kyste radiculaire a été estimé à environ $5 \mathrm{~mm}$ par an [8].

Au cours de leur évolution les kystes radiculodentaires, comme tous les autres kystes des maxillaires, passent par 4 stades $[2,9]$ :

- phase de latence : souvent asymptomatique. Si des signes de desmodontite ne donnent pas l'éveil, c'est la radiologie de la dent mortifiée qui révèle la lésion. Les phénomènes douloureux ne surviennent qu'à l'occasion d'une exacerbation du processus inflammatoire ;

- phase de déformation : marquée par une soufflure de la table externe due à l'augmentation progressive du volume kystique. 
La muqueuse de recouvrement est généralement d'aspect normal ;

- phase d'extériorisation : ou cliniquement, une masse dépressible est palpable. Elle est fluctuante et indolore. La ponction ramène un liquide jaune clair, citrin, parfois hématique, brun chocolat, contenant des paillettes de cholestérol ;

- phase de fistulisation : la table osseuse étant lysée, il ne subsiste plus que la muqueuse qui s'amincit progressivement jusqu'à la fistulisation. La fistule donne issue à un liquide séreux ou séro-hématique.

Radiologiquement (fig. 1 a et fig. $\mathbf{2}$ a), ces kystes se présentent comme une géode homogène, de forme ronde à ovalaire et qui siège au niveau apical ou latéro-radiculaire. La dent causale peut présenter une restauration ou une carie dentaire profonde, une fracture,

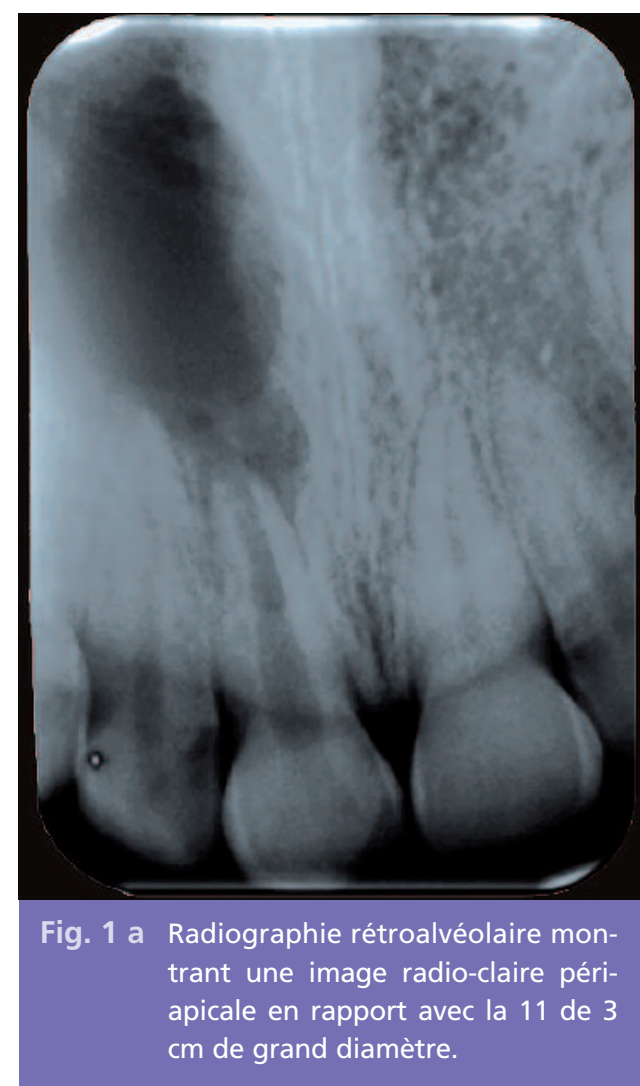

une calcification du canal pulpaire, un traitement endodontique défectueux, comme elle peut être intacte et nécrosée à la suite d'un ancien traumatisme dentaire [10]. Lorsque les lésions augmentent de taille, des rapports de contiguïté s'établissent avec les apex des racines voisines, rendant parfois difficile la détermination de l'apex de la dent causale, ce qui implique de tester la sensibilité de toutes les dents de la région considérée [3]. Également, la lamina dura disparaît au niveau du kyste qui apparaît comme s'il était en continuité avec l'espace desmodontal dentaire. Une résorption des dents voisines peut être retrouvée mais n'est pas habituelle [8]. Les phénomènes inflammatoires aigus estompent en partie ou en totalité le liséré radio-opaque périphérique. Cependant, il reste toujours possible de différencier entre la lésion ostéo-

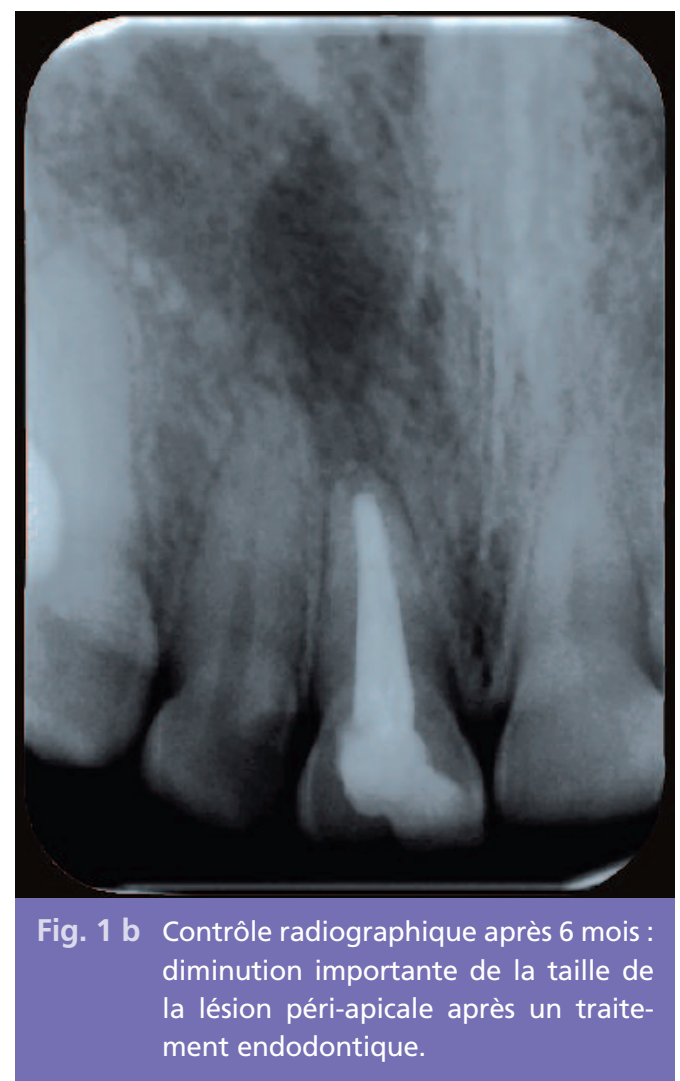


lytique et l'os normal alentour [11]. La radiographie ne permet en aucun cas de faire la distinction entre les différentes formes histologiques des lésions apicales chroniques à savoir : granulome, épithélio-granulome, kyste vrai ou encore kyste en poche. L'examen anatomopathologique est le seul moyen permettant de donner le diagnostic histologique exact $[6,12]$.

Histologiquement [6], un kyste radiculo-dentaire est défini par différents composants. La cavité kystique contient des débris nécrotiques et de cristaux jaunâtres de cholestérol dans 29 à $43 \%$ des cas. Cette cavité est bordée par un épithélium pavimenteux stratifié rarement kératinisé, dont l'épaisseur varie d'un kyste radiculaire à un autre et même d'une région kystique à une autre. Deux types de kystes apicaux sont distingués en fonction de la continuité ou non de leur paroi épithéliale. Il s'agit du kyste en poche qui présente une enveloppe épithéliale ouverte et en continuité avec le canal radiculaire et du kyste vrai qui représente une cavité kystique totalement close $[1,6,12]$.

Sa formation semble pouvoir se faire à partir d'un kyste en poche. Plus la lésion est importante, plus la probabilité d'évolution d'un kyste en poche en kyste vrai est élevée. Le tissu périphérique est infiltré notamment par des macrophages, des lymphocytes, des plasmocytes et plus rarement par des polynucléaires neutrophiles. L'ensemble est contenu dans une capsule fibreuse collagénique [11].

\section{Attitudes thérapeutiques}

Les granulomes, épithélio-granulomes et les kystes en poche, sont susceptibles de guérir dans la mesure où la source de la contamination, à savoir l'infection endodontique, est

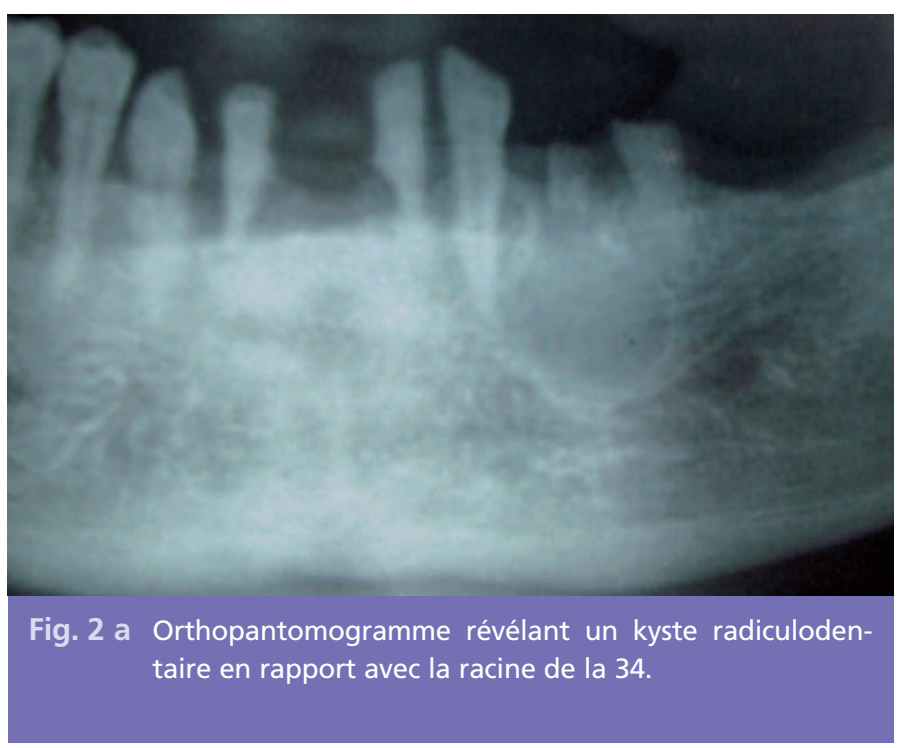

jugulée par une thérapeutique endodontique orthograde adéquate (fig. 1). Les kystes vrais, qui évoluent sans communication avec le système canalaire de la dent concernée, ne peu-

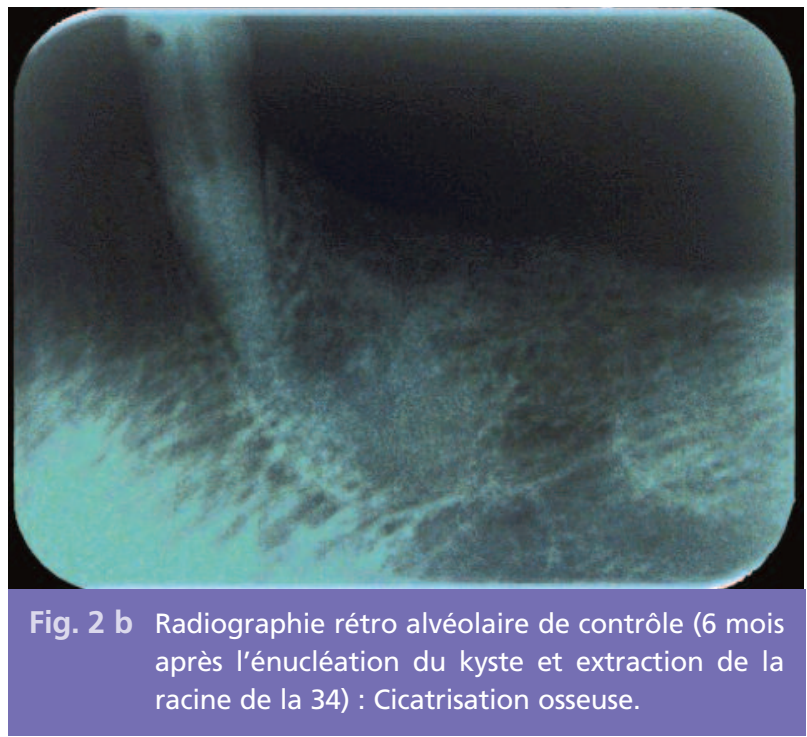


vent pas guérir par un traitement endodontique conventionnel, et la chirurgie endodontique s'impose.

Des études histopathologiques incluant la distinction entre les différentes classes des lésions périapicales chroniques ont montré que, parmi toutes les lésions, la prévalence des kystes vrais reste inférieure à $10 \%$. Ceci n'est pas sans conséquence sur l'approche thérapeutique à adopter. En effet, du moment que plus de $90 \%$ des lésions périapicales chroniques sont susceptibles de guérir par une thérapeutique canalaire conventionnelle, il convient de réaliser un traitement endodontique correct, suivi d'une période de temporisation $(6,9$, 12 mois...), durant laquelle sera instaurée une surveillance clinique et radiologique du cas concerné afin de juger de la réparation ou non de la lésion périapicale [4, 12]. La thérapeutique endodontique correcte consiste en : la désinfection optimale du système endodontique, mise en forme canalaire conique respectant la trajectoire canalaire initiale et préservant les tissus périapicaux, obturation canalaire tridimensionnelle étanche, qui aura pour objectif de neutraliser la totalité du système endodontique et la restauration coronaire définitive hermétique et occlusive.

À l'issue de la thérapeutique endodontique, la guérison peut être obtenue. Les macrophages et autres phagocytes éliminent les tissus altérés y compris les épithéliums en cours de désintégration des parois des kystes et fistules. Les macrophages et les lymphocytes produisent des médiateurs chimiotactiques qui conduisent les fibroblastes jusqu'au foyer inflammatoire. De plus, ils produisent des facteurs activateurs de fibroblastes qui amènent ces cellules à proliférer et à élaborer du collagène pour remplacer les fibres détruites pendant la phase destructive de l'affection. De nouveaux cémentoblastes se différencient et du cément $s^{\prime}$ appose dans les zones de résorption radiculaire. Les ostéoblastes vont synthétiser du tissu osseux qui va remplacer celui qui a été résorbé, ainsi le parodonte périapical est progressivement restauré. Seule une forme irrégulière de l'apex, due au fait que la dentine résorbée n'est pas remplacée, permet un diagnostic rétrospectif de parodontite apicale chronique antérieure $[4,13]$.

La cinétique de guérison des lésions périapicales est variable. Ainsi, après une année, $89 \%$ des lésions susceptibles de guérir manifestent déjà des signes de réparation (réduction de la taille de la lésion), mais uniquement $50 \%$ de ces lésions guérissent totalement en un an. À deux ans, la majorité des lésions guérissent ou continuent à manifester des signes de réparation par réduction de la taille de la lésion. Occasionnellement, cette réduction peut se poursuivre sur 4 ou 5 ans. Les jeunes patients sont d'un meilleur pronostic que les patients plus âgés. Devant les cas d'échecs, à priori initialement, non identifiables cliniquement, le seul recours thérapeutique, après l'endodontie orthograde, reste la chirurgie endodontique. Le pourcentage de succès des traitements endodontiques réalisés sur dents porteuses de lésions périapicales est considérable, il varie de $71 \%$ à $86 \%$ [3].

Cependant, dans le cas d'une lésion en rapport avec une dent délabrée (fig. 2) ou très mobile, le traitement consiste en l'extraction de la dent causale et l'énucléation de la lésion [14]. Pour les kystes volumineux (supérieurs à $200 \mathrm{~mm}^{3}$ ), proches des structures anatomiques nobles (sinus, fosses nasales et canal dentaire) (Progrel, 2004, 2007), cette chirurgie d'énucléation peut être d'une part risquée pour ces structures anatomiques (communication, hypoesthésie), mais également délabrante 
pour les parois osseuses essentielles à l'optimisation de la cicatrisation osseuse donc le trai-

\section{Kyste résiduel}

Le kyste résiduel est défini comme étant un kyste inflammatoire radiculo-dentaire. II subsisterait ou se développerait après une extraction dentaire, soit à partir d'un tissu kystique résiduel, soit à partir de débris épithéliaux non curetés. En effet, aucun document dans la littérature ne fournit de preuve évidente biologique ou clinique du développement d'un kyste résiduel [11].

Ce kyste représente de 2 à $10 \%$ des kystes dentaires [11]. II touche plus fréquemment la femme que l'homme (sexe ratio 3/2). L'âge de survenue est généralement supérieur à 20 ans avec un âge moyen de 52 ans. Le maxillaire supérieur est sa localisation la plus fréquente [10].

Radiologiquement (fig. 3 a), ce kyste apparaît comme une géode. La taille kystique dépasse rarement un diamètre supérieur à $0,5 \mathrm{~cm}$ mais peut être, dans certains cas, suffisamment tement dans ce cas consiste en décompression suivie ou non d'une énucléation.

large pour entraîner une expansion osseuse et une asymétrie faciale [10]. L'aspect histologique du kyste résiduel est identique à celui des kystes radiculaires $[2,8]$.

II persiste une controverse quant à son évolution. Pour certains, ce kyste peut évoluer pour son propre compte, pour certains disparaître progressivement, ce que d'autres contestent.

Les travaux qui ont montré qu'il y avait des kystes vrais, dont la membrane continue était à distance des apex, et des kystes ouverts dont la membrane s'interrompait aux apex, ont permis d'avancer l'hypothèse que l'extraction d'une dent présentant un kyste ouvert laisse la cavité kystique béante, comparable à une sorte de "marsupilisation endoalvéolaire ", qui en décomprimant le kyste le fait régresser. Pour le kyste vrai, si la membrane était à distance de l'apex et qu'elle n'ait pu être lésée par les manœuvres d'extraction et si à fortiori

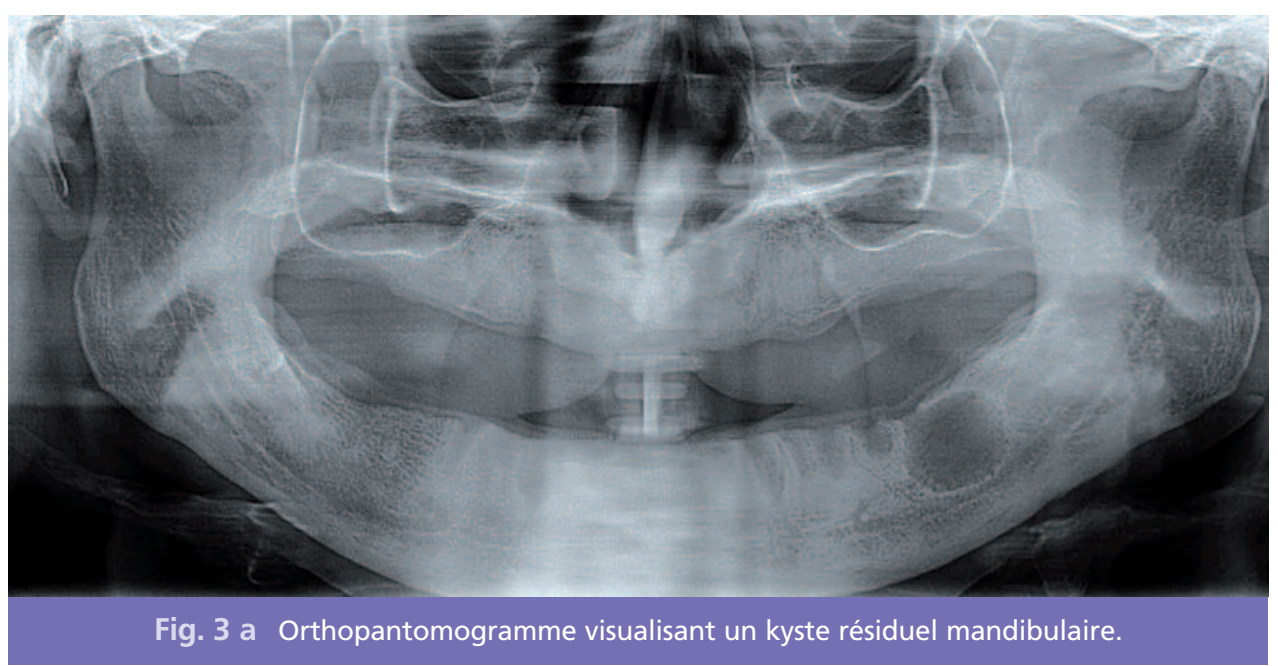


le kyste n'a pas été cureté, il peut évoluer dans le sens d'un développement autonome jusqu'à sa phase d'extériorisation [1].

Une étude de Nair, 1993 [11], met l'accent sur l'impact des cristaux de cholestérol. Ceux-ci précipitent à partir de cholestérol relargué, lors de la désintégration des érythrocytes et des cellules de défense. Les cristaux provoquent une réaction à corps étranger dans le périapex, les cellules géantes étant incapables de les détruire. Selon Nair, I'accumulation des dépôts de cholestérol serait suffisante pour entretenir une lésion.

L'option thérapeutique est en fonction de l'âge, de l'état général du patient ainsi que de la taille kystique [10]. On peut toutefois dire que, pour les formes peu volumineuses, l'attitude est l'énucléation (fig. 3). Dans le cas des kystes résiduels étendus, la décompression avec énucléation subséquente, permettant de ménager les structures vitales du voisinage, constitue également une autre option thérapeutique.
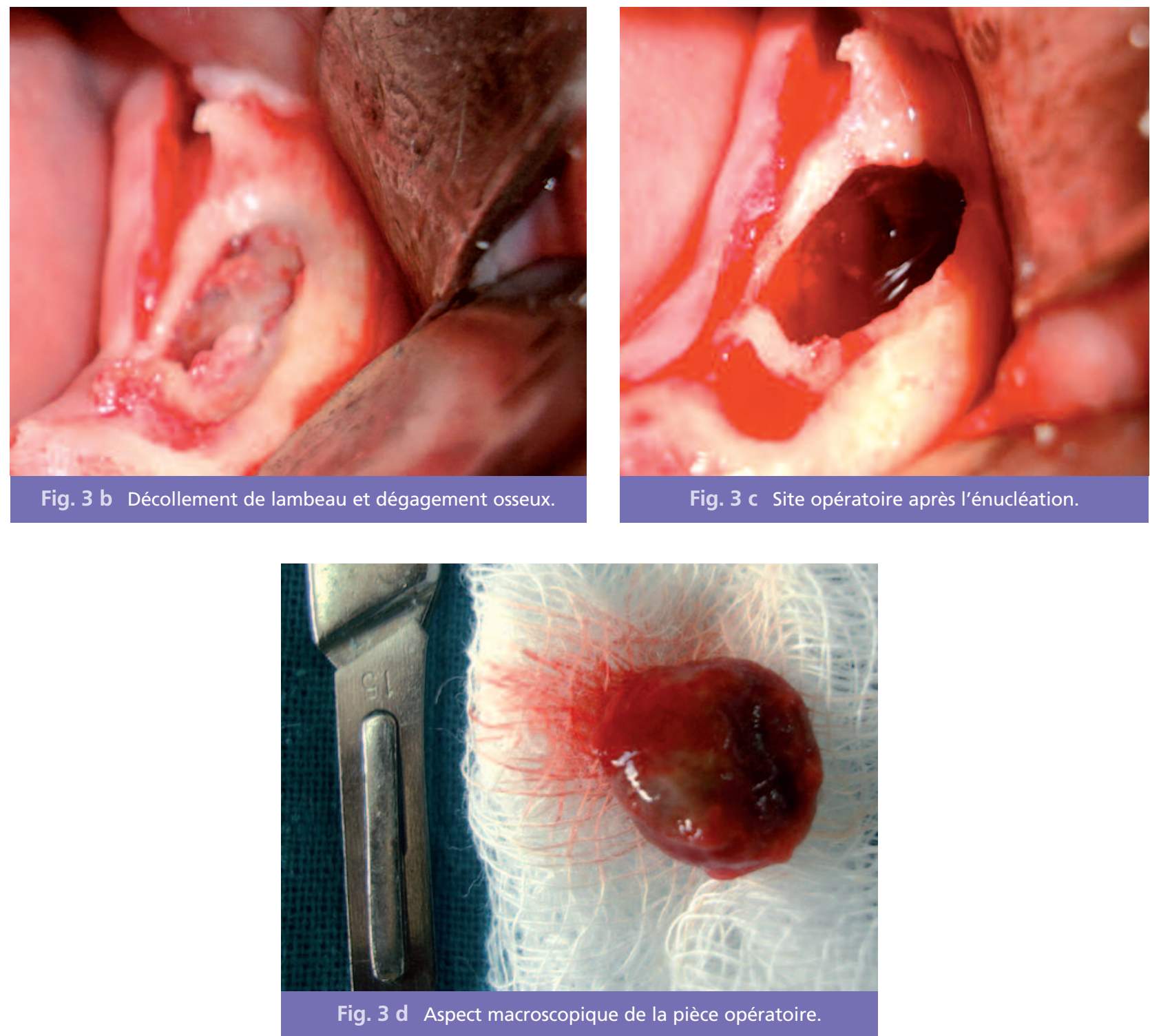


\section{Kyste paradentaire}

Le kyste paradentaire est une variété de kyste odontogène d'origine inflammatoire identifié en 1970 par Main et décrit en 1976 par Craig. Ce kyste n'a été introduit dans la classification histologique internationale des tumeurs odontogènes qu'en 1992 [15].

Cette variété kystique regroupe le kyste inflammatoire collatéral qui se développe en position distale ou vestibulaire d'une dent de sagesse mandibulaire vivante en cours d'éruption (fig. 4) et le kyste vestibulaire mandibulaire infecté qui siège sur la surface radiculaire vestibulaire de la première ou la deuxième molaire et qui affecte essentiellement des enfants âgés de 6 à 8 ans [15].

Le kyste paradentaire représente 0,9 à $4,7 \%$ des kystes odontogènes. La dent de sagesse mandibulaire, souvent enclavée, est sa localisation la plus fréquente, suivie par la $1^{\text {re }}$ puis la $2^{\mathrm{e}}$ molaire permanente mandibulaire et à l'exception des incisives et des canines maxil- laires $[15,16]$.

L'épithélium adamantin réduit les débris épithéliaux de Malassez et les restes de la lame dentaire stimulée par l'inflammation sont supposés jouer un rôle dans la pathogenèse des kystes paradentaires [16].

Diagnostiqué au moment de l'éruption de la dent ou peu de temps après, le kyste paradentaire s'accompagne fréquemment de signes inflammatoires : tuméfaction sensible ou douloureuse, parfois rénitente et de volume modéré, développée sur le versant vestibulaire de la corticale alvéolaire. La vitalité de la dent est constamment respectée, la percussion de l'organe est indolore et il n'existe pas d'atteinte parodontale [15]. L'image kystique est radioclaire, bien délimitée par un liséré d'ostéocondensation périphérique dense. La hauteur du kyste est variable, recouvrant le tiers supérieur des racines ou dépassant largement leurs limites en déplaçant éventuellement les

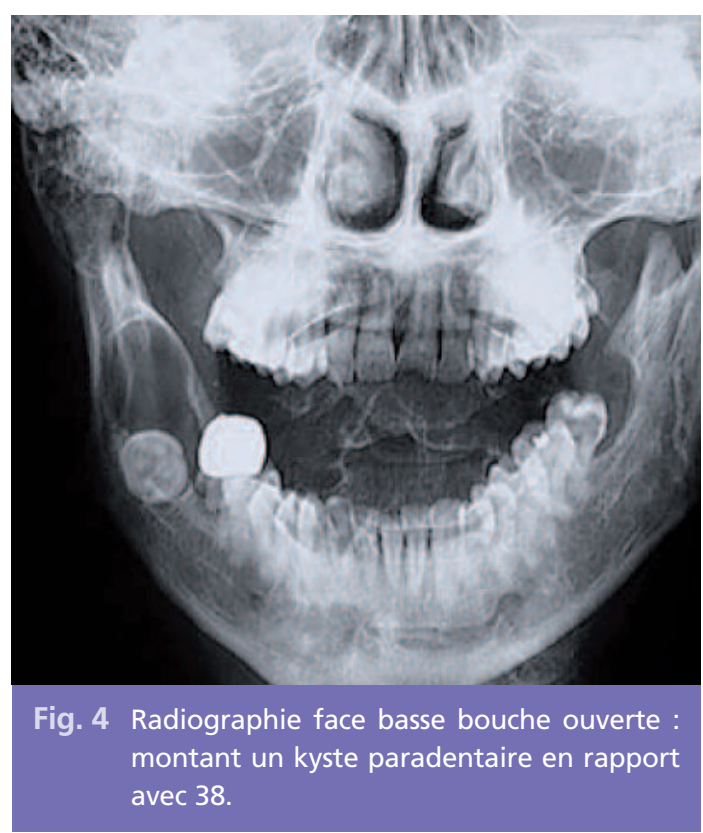


racines dentaires ou les germes dentaires adjacents. Sur les incidences occlusales, le kyste se développe en position distale ou vestibulaire (fig. 4), exceptionnellement mésiale [15].

Au plan histopathologique, l'aspect observé est strictement superposable à celui des kystes radiculaires.
La guérison complète, sans récidive ultérieure, est acquise dès lors que I'on réalise une énucléation du kyste paradentaire et une avulsion de la dent concernée (le plus fréquemment la dent de sagesse); s'il s'agit d'une première ou d'une deuxième molaire, elles seront conservées [14].

\section{Bibliographie}

1. Sauveur G, Ferkdadji L, Gilbert E, Mesbah M.

Kystes des maxillaires. Encycl Méd Chir

(Elsevier SAS, Paris), Stomatologie, 2006;22-062-G-10, Médecine buccale, 2008;28-550-G-10.

2. Benyahya I, Bouachrine F. Aspect cliniques et anatomopathologiques des kystes odontogènes. Le Courrier du Dentiste, janvier 2002.

3. Lasfargues JJ. Le diagnostic clinique des parodontites apicales. Réalités Cliniques 2001;12(2):149-62.

4. Sakout M, El Mohtarim B, Abdallaoui F.

Guérison d'une importante lésion périapicale après traitement endodontique. Le Chirurgien Dentiste de France 2007;1328:41-6.

5. Cochet-Barril I, Simon S. L'hydroxyde de calcium est-il toujours d'actualité ? Les Cahiers de I'ADF 2003;6(16):17-25.
6. Lasfargues JJ, Machtou P. Pathogenèse des lésions périapicales. Réalités Cliniques 2001;12(2):139-48.

7. Laurichesse J.M, Maestroni F, Breillat J.

Endodontie clinique. Paris : CdP, 1986.

8. Piette $\mathrm{E}$, Reychler $\mathrm{H}$. Pathologie des maxillaires. In: Traité de pathologies buccales et maxillofaciales. Bruxelles : De Boeck Université, 1991:1231-4.

9. Le Breton G. Traité de sémiologie et clinique odonto-stomatologique. Paris : CAP, 1997.

10. Wood NK, Goaz PW. Differential diagnosis of oral and maxillofacial lesions. $5^{\text {th }}$ ed. St-Louis: Mosby-year book Inc., 1997.

11. Amor $\mathrm{E}$. Démarche diagnostique face à une lésion radioclaire maxillaire.

Réalités Cliniques 2003;14(1):51-7.

12. Pertot WJ.
Peut-on guérir une lésion périapicale ? Les Cahiers de l'ADF 1999;6:22-7.

13. Tronstad L.

Endodontie clinique. Paris : Flammarion, 1993:32-60.

14. Ruhin B, Guilbert $F$, Bertrand JC. Traitement des kystes, tumeurs et pseudotumeurs bénignes des maxillaires. Encycl Méd Chir (Elsevier SAS, Paris), Stomatologie, [22-062-K10], 2005;1:42-59.

15. Favre-Dauvergne $E_{\text {, }}$ Fleuridas G, Lockhart R, Auriol M, Le Charpentier $Y$, Bertrand JC, Guilbert F. Le kyste paradentaire. Rev Stomatol Chir Maxillofac. 1996;97(1):7-11.

16. Philipsen HP, Reichart PA, Ogawa I, Suei Y, Takata T. The inflammatory paradental cyst: a critical review of 342 cases from a literature survey, including 17 new cases from the author's files. J Oral Pathol Med 2004;33(3):147-55. 


\section{SUMMARY}

\section{Inflammatory cysts of the jaws: a review}

Fouzia HAKKOU

Saliha CHBICHEB

Imane ACHOUR

Wafae EL WADY

\section{Keywords}

- inflammatory cysts

- radicular cyst

- residual cyst

- paradental cyst
The jaw cysts are intraosseous lesions of varied etiology and special histological structure which is characterized by the presence of an envelope that can be keratinized or not. The content might be liquid or semi-liquid. Inflammatory cysts are the most frequent among the jaw's cysts. This variety includes the radicular, residual and paradental cysts. Each type of these cysts has its own features. The aim this paper is to present a literature review on the clinical, radiological and pathological aspects of the inflammatory cysts of the jaws.

\section{Une pédagogie remarquable, des dessins exceptionnels !}

Véritable outil de perfectionnement post-universitaire, en orthopédie et en orthodontie, cet ouvrage est un travail de praticien et d'enseignant. II s'adresse aux chirurgiens-dentistes qui souhaitent développer leur connaissance de l'orthopédie et de l'orthodontie.

Le livre propose certains modèles théoriques permettant de mieux comprendre l'installation, au cours de la croissance sous le jeu des fonctions, de la délicate architecture crâniomaxillo-faciale, ainsi que la définition, en forme, en volume et en situation dans l'espace, des éléments qui la composent.

Chaque atteinte de cet équilibre organise un désordre occlusal, bien particulier, qu'il faut apprendre à reconnaître.

Cette découverte de la création des dysmorphies précise les cibles étiologiques et conduit à la mise en place d'une stratégie thérapeutique et raisonnée.

La construction d'une occlusion, en accord avec les capacités mécaniques mieux comprises des structures qui portent les arcades, doit assurer un résultat fonctionnel, donc stable.

L'auteur, Jean Flour a créé le CETO en 1991 (Centre d'Enseignement des Techniques Orthodontiques Orthopédiques et Fonctionnelles). Il y enseigne la formation avec une pédagogie remarquable et originale issue d'un long travail de synthèse, de recherche et de réflexion. Jean Flour est Docteur en Sciences Odontologiques, Docteur d'Université, Spécialiste Qualifié en Orthopédie Dento-Faciale, et Ancien Maître de Conférence des Universités.

ISBN : 978-2-7598-0601-0

216 pages couleur $-99 €$

Collection «Repères»
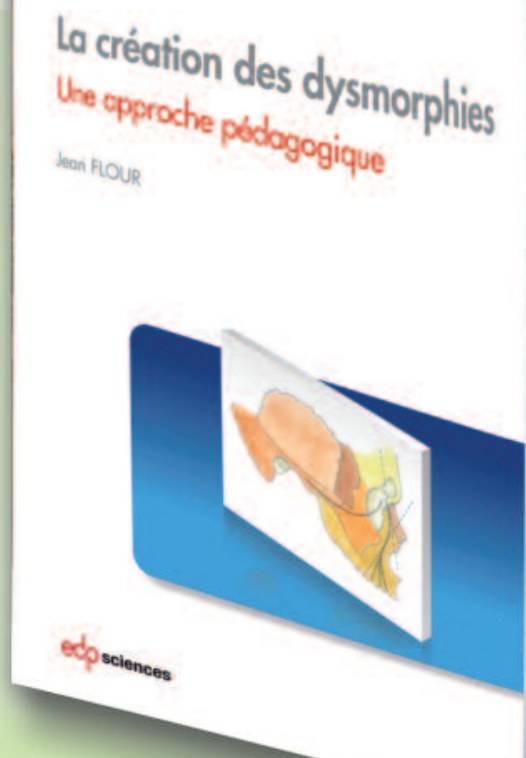